Théologiques

Théologiques

\title{
Quelques recherches récentes en sciences de la nature et leurs incidences théologiques sur la question de l'hominisation1
}

\section{Jean-François Roussel}

Volume 10, numéro 1, printemps 2002

Les animaux dans la conscience humaine

URI : https://id.erudit.org/iderudit/008155ar

DOI : https://doi.org/10.7202/008155ar

Aller au sommaire du numéro

\section{Éditeur(s)}

Faculté de théologie de l'Université de Montréal

ISSN

1188-7109 (imprimé)

1492-1413 (numérique)

Découvrir la revue

Citer cet article

Roussel, J.-F. (2002). Quelques recherches récentes en sciences de la nature et leurs incidences théologiques sur la question de l'hominisation1. Théologiques, 10(1), 27-62. https://doi.org/10.7202/008155ar

\section{Résumé de l'article}

L'orientation actuelle des recherches en paléo-anthropologie ainsi qu'en étude de la cognition animale comporte des implications importantes pour l'anthropologie théologique, plus particulièrement à propos de l'émergence de l'humanité au sein du monde animal. Après avoir résumé l'état de la question à propos de l'hominisation en théologie et les contributions en théologie systématique contemporaine concernant la place de l'être humain dans la nature, puis formulé certaines précisions méthodologiques, l'article présente des recherches récentes (1996-2001) en biologie et en paléo-anthropologie. Ces recherches questionnent l'extension et l'usage du concept d'hominisation, à propos duquel des propositions sont formulées ici à l'usage de la théologie. Enfin, des enjeux théologiques cruciaux se dégagent de l'étude et sont présentés. 


\title{
Quelques recherches récentes en sciences de la nature et leurs incidences théologiques sur la question de l'hominisation ${ }^{1}$
}

\author{
Jean-François RousseL \\ Faculté de théologie \\ Université de Montréal
}

Comment la théologie peut-elle intégrer les recherches les plus récentes à propos de l'émergence de l'être humain, de même que ce que d'autres recherches récentes en cognition animale suggèrent à ce propos? Quelle serait la réponse de la théologie à la question du rapport entre l'humanité et l'animalité que posent les recherches récentes, en biologie et paléo-anthropologie, relativement à l'évolution et l'hominisation? Relayées par les journaux, médias électroniques et publications de vulgarisation scientifique dont il sera fait état ici, ces recherches comportent des incidences anthropologiques et des conséquences théologiques.

Des recherches récentes peuvent rarement prétendre à un statut autre que provisoire. Néanmoins, la théologie, si elle entend vraiment œuvrer en relation avec les sciences de la nature, doit assumer cet aspect provisoire. Il en va de même pour la valeur de chacune des recherches en sciences de la nature considérée individuellement. Notre intention est de dessiner une orientation générale des recher-

1. Je remercie M. Christian Downs, étudiant au Ph.D. en sciences des religions à la Faculté de théologie de l'Université de Montréal, pour sa contribution à la recherche documentaire en vue de produire cet article. 
ches en cours et de relever, pour la théologie, certaines implications de cette orientation, quels que soient les résultats particuliers obtenus de même que leur valeur, considérée au cas par cas. Par ailleurs, les résultats de recherches scientifiques dont il est fait état ici font appel à des méthodologies impossibles à présenter dans l'espace qui nous est imparti, et nous avons conscience de ne présenter ici les choses que sous un jour hautement sommaire. Il est entendu qu'un article théologique ne vise pas à évaluer ces méthodologies. Plutôt, notre projet est de montrer dans quelles directions s'orientent les pistes de recherche actuellement pratiquées, particulièrement en paléo-anthropologie et en étude de la cognition animale, et quels impacts potentiels en résultent pour la théologie.

Nous commencerons par rendre compte de l'état de la question sur l'hominisation en théologie et, de manière élargie, sur les contributions en théologie systématique contemporaine concernant la place de l'être humain dans la nature. Par la suite, nous porterons attention à la question, méthodologique, de l'articulation d'une référence scientifique (au sens de science de la nature, ou dite exacte) dans un discours théologique. Cette question étant répondue de manière à ce que soit bien comprise la manière dont nous articulerons nous-même des données scientifiques à une étude théologique, nous rendrons compte de recherches récentes (1996 et après) en biologie et en paléo-anthropologie. Ces recherches, à notre avis, ne sont pas sans implications de taille pour la théologie chrétienne et pour la manière dont l'anthropologie théologique traite le thème de l'hominisation. Pour montrer ces implications, nous réfléchirons le concept d'hominisation et soumettrons notre thèse à son sujet. De là, nous préciserons les enjeux théologiques qui nous paraissent les plus cruciaux au terme des analyses que nous aurons menées.

\section{1. État de la question en théologie contemporaine}

La question de l'hominisation a connu un retentissement théologique important au siècle dernier. Formulée en lien avec les recherches en paléo-anthropologie, elle est largement redevable aux écrits du pionnier que fut Teilhard de Chardin. De manière correspondante, 
elle a fait l'objet de décrets magistériels au sein de l'Église catholique, le plus retentissant de tous étant Humani generis, sous le pontificat de Pie XII (1950)².

\subsection{Une exploration entre sciences de la nature et théologie: Teilhard de Chardin}

Il convient de s'arrêter quelque peu sur le cas de Teilhard lui-même, dont le destin et la réception posthume restent exemplaires de la méfiance persistante, mais aussi de la séduction qu'il continue à produire sur certains esprits contemporains. Teilhard a été critiqué à la fois du sein de la théologie et de la paléo-anthropologie. Dans le premier champ disciplinaire, outre les "ambiguités » et les «erreurs graves " que le Saint-Office dénonçait dans ses œuvres en 1962 sans les préciser davantage, d'aucun jugèrent que sa pensée ne tenait pas suffisamment compte du problème du mal ${ }^{3}$. Par ailleurs, on a reproché à Teilhard un manque de clarté théologique. À ce sujet, à propos de l'interdiction de publier qui avait frappé le principal intéressé à partir de 1948, Lodovico Galleni n'a-t-il pas raison de

2. Humani generis dénonçait des «erreurs » scientifiques, philosophiques et théologiques, parmi lesquelles l'existentialisme, l'idéalisme, le polygénisme et la «nouvelle théologie» des années 40-50 avec son éloignement de la néo-scolastique et sa relecture historico-critique des sources chrétiennes. Sur la question de l'évolution et de l'hominisation, Pie XII insistait sur le caractère hypothétique de la théorie darwinienne et la mettait en balance avec le caractère assuré de la révélation divine, interdisant dès lors aux catholiques d'adhérer à toute hypothèse qui contredirait celle-ci.

3. À cet égard, on connaît les dernières pages de son Phénomène humain: "Ma réponse [...] est que, attaché dans cet ouvrage au seul dessein de dégager l'essence positive du processus biologique d'hominisation, je n'ai pas cru nécessaire (par raison de clarté et de simplicité) de faire le négatif de l'image que je projetais.» (P. Teilhard de Chardin, Le phénomène humain, Paris, Seuil, 1955, p. 345) Puis Teilhard définit sommairement le mal sous quatre formes, sous-produits inévitables du processus évolutif. Le mal, si tragique soit-il, est l'effet des avancées d'un univers tâtonnant - à charge pour la théologie de repérer quelque cause supplémentaire, ce sur quoi Teilhard déclare: "Je ne me sens loyalement pas en mesure, et ce n'est du reste pas le lieu ici, de prendre position.» (p. 348) 
demander: "Comment obtenir cette clarté alors qu'on refusait précisément aux écrits teilhardiens ce qui en [est] la source fondamentale, à savoir ce développement progressif des idées qui ne peut venir que du libre débat et de la libre confrontation ${ }^{4}$ ? » L'œuvre de Teilhard reste inachevée, une interpellation à la théologie qui n'a pu obtenir de réponse constructive du vivant de son auteur.

Hors de la théologie, tout en rappelant les travaux de Teilhard en paléo-anthropologie (autour de la découverte du sinanthropus en 1929), on ne l'a pas suivi dans ce qu'on a appelé son finalisme. En effet, si l'on suit les modèles théoriques qui ont cours actuellement en sciences de la nature, l'apparition de l'humain ne découle pas d'une nécessité liée à l'organisation cosmique; l'évolution des espèces, si elle opère à partir des seules possibilités génétiques disponibles, obéit toutefois aux principes de la sélection naturelle, de l'adaptation à l'environnement (par essais et sélection dans les possibilités aléatoires qui sont réalisées) et de la physique quantique - ce qui implique une indétermination inhérente à l'organisation des particules. Par conséquent, quant aux lois qui gouvernent les mutations et quant aux espèces qu'elle produit, l'évolution s'effectue comme un jeu ouvert. L'hypothèse finaliste ou téléologique ne franchit pas le test de la vérification: l'évolution comporte une marge d'indétermination indissociable de la possibilité même d'une évolution ${ }^{5}$.

Enfin, en théologie comme en sciences de la nature, on souligne la contribution, substantielle et courageuse, du théologien-paléontologue au rapprochement entre théologie et science. Teilhard a le mérite qui incombe aux éclaireurs, mais aussi leurs lacunes occasionnelles: il formule des intuitions, les théorise, et encourt le risque de la fragilité. Sa pensée n'en continue pas moins à intéresser des esprits venus d'horizons divers ${ }^{6}$.

4. L. Galleni, «Teilhard de Chardin, le message » Concilium, 284 (2000) p. 138.

5. Sur ces questions, voir: I. Prigogine et I. Stengers, La nouvelle alliance. Métamorphose de la science, Paris, Gallimard, 1979 et J. Monod, Le hasard et la nécessité, Paris, Seuil, 1970.

6. Dans une entrevue, en 2001, le professeur émérite d'écologie Pierre Dansereau avoue l'interpellation qu'il reçoit de Teilhard: E. ZEHLER, «Une entrevue avec 


\subsection{L'être humain comme devenir spirituel de la matière: Rahner}

Après sa réduction au silence et son décès en 1955, d'autres reprennent la question de l'hominisation en théologie. Les limites de cet article nous empêchent de présenter en détail les contributions théologiques marquantes des dernières décennies concernant l'hominisation et le statut de l'humain dans la nature. Un survol est néanmoins nécessaire pour situer la théologie actuelle et les déplacements qui semblent suggérés ou appelés par les recherches extra-théologiques en cours.

Pierre Dansereau: Plaidoyer pour une discipline. Pour une lecture du paysage dans sa totalité " dans Le Devoir (samedi, 7 avril 2001) p. E-3. D'autre part, alors que le réseau Internet s'étend rapidement sur la Terre, Teilhard trouve une audience inattendue, qui fait du théologien de la noogénèse le visionnaire d'un nouveau seuil évolutif, où les individus se réunissent pour constituer une forme d'intelligence collective. L'Internet serait-il un nouveau stade de l'hominisation ? Hypothèse intéressante, à trois conditions: d'une part, trouver un moyen de la vérifier, ce qui parait difficile; d'autre part, tenir compte de la thèse de Ganoczy, à l'effet qu'en parvenant au stade humain, l'évolution des espèces intègre des facteurs culturels en plus des facteurs biologiques et génétiques [A. Ganoczy, Dieu, l'homme et la nature. Théologie, mystique, sciences de la Nature, Paris, Cerf, 1995 (allemand 1992), p. 261]. Enfin, placer au rang des épiphénomènes les intérêts financiers qui régissent la "toile", la publicité et le bavardage qui y foisonnent, de même que ses utilisations criminelles. Se pourrait-il que, à l'instar de leur maître, les cyber-teilhardiens errent par excès d'optimisme? Toutefois, à la considérer au ras des phénomènes, l'évolution des espèces ignore les prescriptions morales, et l'accroissement des capacités humaines n'a jamais exclu leurs utilisations médiocres ou aberrantes. Voir parmi une abondante littérature de vulgarisation, P. CHARTiER, «Le grand prêtre du Net ", Québec science, 38/10 (juillet-août 2000) p. 44-45; J.-F. DorTIER, "Vers une intelligence collective?", Sciences humaines, no 32 (mars-avril-mai 2001) p. 22-24; et sous un jour plus critique, S. Allemand, "Gare au culte d'Internet ", Sciences humaines, no 32 (mars-avril-mai 2001) p. 26. Enfin, sans référence à Teilhard et loin de toute téléologie, le paléontologue Claude-Louis Galien conclut son histoire de l'humanité par l'évocation d'un nouveau seuil évolutif possible (à côté de l'extinction ou simplement d'une humanité plus "sage»): la connexion des intelligences humaines entre elles par l'informatique (C.-L. Galien, Homo. Histoire plurielle d'un genre très singulier, Paris, PUF, 2002, p. 431-432). 
Dans un article écrit au temps de Vatican II, K. Rahner aborde la question du monogénisme, de la qualification théologique de la condamnation de cette théorie par l'encyclique Humani generis et des degrés d'obligation et de liberté de la théologie à l'égard de cette condamnation. Il tente une exégèse des passages bibliques référant aux origines de l'humanité. L'article en question est intéressant tout autant par son étude du thème étudié que par l'exemple qu'il constitue d'une herméneutique fine des énoncés doctrinaux, susceptible d'instruire encore aujourd'hui des étudiants en théologie dogmatique. Laissons de côté les nombreux éléments de cet article qui sont sans lien direct à la question qui nous intéresse ici. Parmi ceux-ci, celui qui nous concerne directement concerne l'unité ontologique et historique du genre humain, condition préalable à une compréhension du rapport d'appartenance du Christ à la totalité de l'humanité7. Sur la base d'une exigence de cohérence des doctrines apparentées du péché originel de l'unique Adam et du salut offert en l'unique Christ, Rahner soutient que le monogénisme est une position nécessaire dans une perspective théologique. De plus, Rahner croit qu'adhérer à la thèse polygéniste reviendrait à poser une multiplicité d'origines et, ce faisant, une multiplicité d'espèces à l'intérieur de ce qui nous apparaîtrait comme l'humanité; position intenable, pour les raisons présentées ci-dessus. Enfin, Rahner insiste sur la différence radicale de l'humain , "en un sens strictement métaphysique ", par rapport à toute vie «infra-humaine » : toujours au sens métaphysique, "Il n'est pas une simple combinaison et variation provenant de ce qu'il y a en dehors de lui dans le monde matériel. Ce qu'il est ne peut pas être conçu comme une transformation d'autres réalités ${ }^{8}$.» En effet,

par rapport au monde animal, l'homme est une espèce essentiellement différente, métaphysiquement nouvelle, non seulement au point de vue des types (au sens biologique), non seulement dans le gène, mais dans

7. K. Rahner, «Le monogénisme et la théologie» dans Écrits théologiques, vol. 5, Paris, Desclée de Brouwer, 1966, p. 60-74.

8. RAHnER, «Le monogénisme et la théologie», p. 78. 
la racine suprême de son être psycho-corporel, située derrière la sphère de l'empirie externe, dans sa forme spirituelle? .

Il est à remarquer que Rahner s'en tient ici au niveau précis d'une conception métaphysique des commencements humains, en laissant explicitement ouverte la question de ce «qui est déjà présent dans le monde comme réalité vivante ou non, [qui] puisse être introduit dans ce nouveau commencement originel, [et du] [...] réel enchaînement génétique avec le monde animal que l'homme puisse avoir dans la seule dimension du vital ${ }^{10}$ ».

Par ailleurs, à la question de la place de l'humain dans le cosmos, Rahner a consacré des pages de la section christologique de son Traité fondamental de la foi (205-221). Rahner semble se dissocier ici de l'approche de Teilhard, sans le nommer toutefois. En particulier, pour Rahner, le fait d'intégrer la christologie à la cosmologie revient à faire de la théologie une philosophie, la liberté de l'autocommunication de Dieu se trouvant ici reléguée aux oubliettes. Observons d'ailleurs que l'intégration du thème à la christologie est significative du souci de Rahner: si Teilhard introduisait son Christ cosmique dans une étude cosmologique, Rahner prend le Christ pour cadre d'appréhension du problème de l'hominisation. Si «la théologie est une anthropologie ", cela ne veut pas dire que la théologie soit à la remorque d'une anthropologie qui pourrait être construite en dehors de la théologie ${ }^{11}$. D'autre part, appuyant son anthropologie sur le concept d'autotranscendance active, Rahner définit l'homme

comme l'étant dans lequel la tendance fondamentale de la matière à se trouver elle-même dans l'esprit parvient, par autotranscendance, à sa percée définitive, de telle sorte qu'à partir de là l'essence de l'homme lui-même peut être vue à l'intérieur d'une conception fondamentale et globale du monde ${ }^{12}$.

9. RAHNER, «Le monogénisme et la théologie », p. 83-84.

10. RAHner, «Le monogénisme et la théologie», p. 78-79.

11. La fameuse expression de 1968 est donc à entendre à l'encontre de ce que les adversaires de Rahner lui avaient reproché, au premier chef Hans Urs von Balthasar.

12. Rahner, "Le monogénisme et la théologie", p. 208. 


\subsection{Après Rahner: Sciences de la nature, écologie et éthique}

Nous aurons l'occasion de revenir sur cette approche de Rahner. Un peu plus récemment, dans une conceptualité et à partir de préoccupations historiques fort différentes, un ouvrage de Jürgen Moltmann en théologie de la création a fait date ${ }^{13}$. En pleine guerre froide, il propose une théologie écologique de la création, qui situe l'humain dans la chaîne des créatures vivantes, dont il est responsable ${ }^{14}$; Moltmann prolonge ici l'important article de Gerhard Liedke de 1980 sur le fameux verset de la Genèse: «Faisons l'homme à notre image, selon notre ressemblance et qu'il soumette les poissons de la mer, les oiseaux du ciel, les bestiaux, toute la terre et toutes les petites bêtes qui remuent sur la terre ${ }^{15}$.» (Gn 1,26) Alexandre Ganoczy, de son côté, s'est appliqué à articuler les perspectives de la théologie et des sciences de la nature, en une synthèse qui impressionne particulièrement par sa culture scientifique: s'y entrecroisent en effet, à côté des perspectives de la philosophie et de la tradition théologique chrétienne, celles de la paléo-anthropologie, de la physique quantique et des neurosciences ${ }^{16}$. Du côté américain, Gordon

13. J. Moltmann, Dieu dans la création. Traité écologique de la création, trad. par M. Kleiber, Paris, Cerf, 1988 (allemand 1985).

14. Sur le thème de la responsabilité, on songe à H. JonAs, Le principe responsabilité. Une éthique pour la civilisation technologique, traduit par Jean Greisch, Paris, Cerf, 1990 (allemand 1979). D’autres que Moltmann citent la responsabilité comme trait d'humanité: J.-M. Maldamé, "Nouveau regard sur l'émergence humaine », Bulletin de littérature ecclésiastique, 94/4 (1993) p. 339; A. Ganoczy, Dieu, l’homme et la nature, p. 261; G. Lepoutre, «Émergence dans l'univers, émergence de l'humanité ", Mélanges de sciences religieuses, 55/1 (1998) p. 23.

15. G. Liedke, «La solidarité dans le conflit. Le créateur, la création et la créature ", dans J. L. Blondel (dir.), Science sans conscience, Genève, Labor et Fides, 1980, p. 47-57.

16. Ganoczy, Dieu, l'homme et la nature, en particulier les p. 249-273 sur l'évolution cosmique, celle des espèces et l'hominisation. Sa Théologie de la nature est d'un abord plus accessible. L'auteur n'y traite que peu de l'hominisation, mais propose une théologie biblique de la nature: A. Ganoczy, Théologie de la nature, trad. par J. Doré et C. Flamant, Paris, Desclée, 1988 (allemand 1982). 
Kaufman et Sallie McFague ont traité l'hominisation et le rapport de l'humain à l'animal dans un souci d'opérativité sociale des concepts théologiques, le premier dans le cadre général de son entreprise de reconstruction des concepts théologiques, la seconde dans la veine de l'éco-féminisme, sous la double influence de Kaufman et de la théologie Process ${ }^{17}$. Citons enfin le Britannique Arthur Peacocke, dont il sera question plus loin dans cet article: s'il réfère de manière informée aux recherches en sciences de la nature, son entreprise trahit un souci d'ordre apologétique : comment concilier la foi chrétienne en un Dieu créateur, tel qu'exprimée dans la Bible, et les résultats de ces recherches ${ }^{18}$ ?

En résumé, dans sa manière de penser l'hominisation et le rapport de notre espèce au reste du monde animal, le corpus écrit après Rahner et avant 1996 évolue dans le cadre général de deux interpellations: celle, descriptive et scientifique, venue de la physique, de la paléontologie, de la paléo-anthropologie ; l'autre, éthique, dans l'horizon de la crise écologique.

Quelques traits rapprochent ces écrits, outre le fait qu'ils postulent tous la validité du «récit commun de la création », défini selon

17. G.D. Kaufman, In Face of Mystery: A Constructive Theology, Cambridge, Mass. / London UK, Harvard University Press, 1993; S. McFague, The Body of God: An Ecological Theology, Minneapolis, Fortress 1993.

18. A. Peacocke, Theology for a Scientific Age: Being and Becoming - Natural, Divine, and Human, Minneapolis, Fortress Press, 1993. Pour fins de mémoire, ajoutons: M. Fox, Original Blessing: A Primer in Creation Spirituality, Santa Fe, Bear \& Co, 1983; A. Gesché, "Cosmologie et anthropologie », dans Dieu pour penser, 4: Le cosmos, Paris, Cerf, 1994, p. 23-47; P. Gisel, La création. Essai sur la liberté et la nécessité, l'histoire et la loi, l'homme, le mal et Dieu, Genève, Labor et Fides, 1980; W.J. Houston, " "And let them have dominion...” Biblical Views of Man in Relation to the Environmental Crisis », dans E.A. Linvingstone, dir., Studia Biblica 1978 (Journal for the study of the Old Testament. Supplement series 2), Sheffield, JSOT Press, 1979, p. 161-184; H. Kessler, Das Stöhnen der Natur. Plädoyer für eine Schöpfungsspiritualität und Shöpfungsethik, Düsseldorf, Patmos Verlag, 1990; R.R Ruether, Gaia and God: An Ecofeminist Theology of Earth Healing, San Francisco, Harper \& Row, 1992; G. Siegwalt, "L'actualité de Gn 1», Revue d'Histoire et de Philosophie Religieuse, 59 (1979) p. 319-325. 
les balises des sciences de la nature ( «common creation story », par opposition au «classic organic model $\left.^{19} »\right)$. Une question est commune: quelle est la pertinence d'une théologie de la création et de l'émergence humaine, au regard des positions actuelles des sciences de la nature, après Darwin en particulier?

D'autres problèmes sont aussi partagés: celui de la finalité des processus évolutifs, qui questionne la notion théologique de télos divin et d'histoire du salut: l'humanité correspond-elle au dessein d'une intelligence créatrice ou relève-t-elle plutôt du hasard, comme on pourrait le déduire dans la foulée de Monod? La physique quantique, qui se développe autour d'une indétermination inscrite dans la matière elle-même, invite à penser l'émergence humaine à partir du chaos et du hasard. Or, l'aléatoire de l'hominisation demande à être pensé sur le plan théologique. En ce sens, délaissant le finalisme teilhardien, Ganoczy et Moltmann, le second en particulier, pensent l'indétermination de l'émergence humaine comme «jeu divin». La contingence, au sens du contraire de la nécessité, est inscrite dans l'humanité, à son origine. L'humain serait donc une créature comme les autres? Une espèce apparue de façon hasardeuse (et qui pourrait un jour céder sa place à une autre, non humaine mais plus évoluée) ? Sur ce point, Jean-Michel Maldamé, proche ici de Gérard Lepoutre, pense l'émergence humaine à partir d'une conceptualité systémique: il ne définit pas l'humain a priori, mais en fonction d'une critériologie valable pour tous les organismes vivants. Pourtant, "l'homme est humain par l'actualisation de son potentiel génétique, biologique et écologique. Il en a la responsabilité. ${ }^{20}$ " Par le langage, la main, le visage, la sexualité, ce potentiel est actualisé et dès lors, «le corps humain n'est pas placé à l'intérieur d'une chaîne de vivants homogènes, car les actes qui caractérisent la vie sont le fruit d'une humanisation, qui se fait à partir de l'humain, de l'âme ${ }^{21}$ ». L'hominisation est aléatoire, mais résulte en une créature singulière, qui ne fait plus série avec celles qui l'ont précédée.

19. McFague, The Body of God, p. 30s.

20. Maldamé, «Nouveau regard sur l'émergence humaine ", p. 339.

21. Maldamé, «Nouveau regard sur l'émergence humaine», p. 339. 
Ces théologies posent aussi les problèmes reliés de l'appartenance de l'humanité au monde vivant et de ce qui la spécifie. Le refus d'un anthropocentrisme exclusiviste se conjugue à la mention de traits propres à l'humain: langage et conscience réflexive, par Moltmann et McFague, et caractérisation théologique de l'humain comme créé à l'image de Dieu (Moltmann en particulier). De ces caractéristiques, plusieurs infèrent la responsabilité de l'humain à l'égard de la biosphère: s'il est partie prenante du règne animal, il en constitue l'espèce la plus consciente ${ }^{22}$. On rejoint ici un autre enjeu, présent chez Liedke, Kaufman, McFague et Moltmann, celui de la pertinence de Gn 1,26.28 dans le contexte de la crise écologique et des possibilités techniques qui l'ont rendue possible.

Enfin, ces théologies traitent la question du rapport entre immanence et transcendance de Dieu dans l'univers; on perçoit ici les échos de la condamnation de Teilhard, pour cause de panthéisme larvé, et la proximité de la théologie process, surtout chez McFague. Ainsi, Ganoczy alerte contre ce panthéisme possible et propose plutôt de penser l'évolution sous le mode théologique en référence au concept rahnérien d'autotranscendance active; Moltmann, pour sa part, insiste sur la nécessaire polarité de la transcendance et de l'immanence; et Peacocke distingue panthéisme et panenthéisme, où la nature jouerait pour Dieu une fonction analogue au corps pour les êtres humains ${ }^{23}$.

\section{Précaution méthodologique}

L'examen de la littérature théologique autour de l'hominisation et du "récit commun de la création» ( «common story of creation») montre un désir de "réenchanter» l'univers, selon l'expression de Bernard Micholet, elle-même inversion d'un concept fameux de Marcel Gauchet. Sans doute la théologie peut-elle à bon droit s'as-

22. Voir M.E. Clark, "Human nature: What we need to know about ourselves in the twenty-first century», Zygon, 33/4 (1998) p. 645-658.

23. Ganoczy, Dieu, l'homme et la nature, p. 268-270; Moltmann, Dieu dans la création, p. 266; Arthur PeAcocke, Theology for a Scientific Age, p. 708. 
signer un projet de cet ordre. Comment procède-t-on alors? Un passage d'Arthur Peacocke est exemplaire par la manière dont il raconte l'évolution cosmique en un style qui rappelle $\mathrm{Gn} 1^{24}$ :

Dieu était. Et Dieu était Tout-ce-qui-était. L’amour de Dieu déborda, et Dieu dit: "Que l'Autre soit. Et qu'il possède la capacité de devenir ce qu'il pourrait être, et ce faisant se faisant lui-même. Et qu'il explore ses potentialités. » Et il y eut l'Autre en Dieu, un champ d'énergie, d'énergie vibrante - mais ni matière, espace, temps ni forme. Obéissant à la loi qu'il avait reçue, et avec un surgissement d'énergie intensément chaud - un big bang brûlant - cet Autre explosa en univers, il y a 12 milliards d'années (plus ou moins), créant ainsi l'espace.

Tourbillonnante, la matière fondamentale apparut, s'étendit et s'étendit, et refroidit en nuages de gaz, en un bain de lumière irradiante. L'univers continua à étendre et à se condenser en tourbillons de matière et de lumière, en un milliard de galaxies.

Il y a cinq milliards d'années dans une galaxie, une étoile - notre Soleil — réunit de la matière autour d'elle sous forme de planètes. L'une d'entre elles était notre Terre. Sur Terre, masse atomique et température atteignirent le seuil requis pour permettre à l'eau et au roc de former des continents, et les montagnes surgirent. Et en quelque crevasse humide, où bassin, ou dans les profondeurs de la mer, il y a plus de trois milliards d'années, des molécules devinrent assez grosses et complexes pour produire des copies d'elles-mêmes et devenir les premiers points de vie.

La vie se multiplia dans les mers, se diversifiant et devenant de plus en plus complexe. Il y a 500 millions d'années, apparurent des créatures dotées de squelettes solides, les vertébrés. Sur la terre ferme, les plantes vertes transformèrent l'atmosphère en créant de l'oxygène. Puis, il y a 300 millions d'années, certains poissons apprirent à ramper en dehors de la mer et à vivre sur les rives, respirant l'oxygène de l'air.

Alors la vie éclata sous plusieurs formes - reptiles, mammifères (et dinosaures) sur la terre ferme, reptiles et oiseaux dans les airs. Au cours de millions d'années, les mammifères commencèrent à développer des cerveaux complexes qui leur permirent d'apprendre. Certains d'entre

24. Dans le présent article, toutes les traductions françaises des textes de langue anglaise cités en bibliographie sont de nous. 
eux habitaient dans les arbres. Nos premiers ancêtres naquirent d'eux puis, il y a 40000 ans, le premier hommes et la première femme apparurent. Ils commencèrent à connaître des choses à propos d'euxmêmes et des gestes qu'ils posaient; non seulement étaient-ils conscients, mais en outre ils étaient conscients d'eux-mêmes. On entendit le premier mot, le premier rire. On créa les premières peintures. On eut la première intuition d'un au delà, ainsi que les premiers signes d'espoir à ce sujet - en effet, ils enterraient rituellement leurs morts. On fit les premières prières à Celui qui avait fait Tout-ce-qui-est et Tout-ce-qui-devient. On fit les premières expériences du bien, de la beauté et de la vérité, mais aussi de leurs contraires, les humains étant libres ${ }^{25}$.

Comme une large part des sources examinées au cours de la préparation du présent article, ce récit dénote un passage d'une théorie scientifique, celle du Big Bang, à une narration métaphysique. Ici comme dans le reste de l'article dont ce passage est tiré, Peacocke entreprend de déduire un sens théologique et métaphysique d'une thèse scientifique. Or, la science évolue dans l'ordre du provisoire et porte sur des phénomènes ou calculs délimités. D'une position scientifique, largement admise de nos jours par ailleurs, l'auteur dégage un mythe, au sens que donna un jour Lévy-Strauss à ce mot: "Le propre du mythe, c'est confronté à un problème, de le penser comme l'homologue d'autres problèmes qui se posent sur d'autres plans: cosmologique, physique, moral, juridique, social, etc. ${ }^{26}{ }$ À tout le moins, Peacocke élargit la portée du big bang à l'éthique et à l'esthétique, en parlant de la beauté, de la liberté, du bien et de leurs contraires.

Bernard Micholet met en garde contre un détournement de la science vers le projet d'un grand récit dont l'émergence n'aurait rien à voir avec les méthodes scientifiques mais qui s'en tiendrait à utiliser la science comme bassin de matériaux éventuellement «mythologisables». La théologie systématique n'est pas une science empirique. Par ailleurs, elle ne doit certainement pas s'interdire d'ac-

25. PeAcocke, «Biology and a Theology of Evolution", p. 696.

26. C. Lévy-Strauss et D. Eribon, De près et de loin, Paris, Odile Jacob, 1988. 
cueillir les données des recherches en sciences de la nature. Ce qu'elle devrait toutefois se garder de faire, c'est de chercher à fonder sa compatibilité avec des données scientifiques en détachant celles-ci de leurs présupposés empiriques et expérimentaux, pour les transposer en énoncés à portée métaphysique et ontologique. Plus positivement, la théologie, quand elle entend intégrer les données de la science, doit accepter d'avancer dans l'ordre d'une énonciation hautement provisoire et de composer avec ce que les données souvent provisoires des sciences de la nature proposent à la pensée.

Nous tenterons, bien sûr, de nous conformer nous-même à ces deux règles. Aussi les résultats de recherches présentés dans le présent article n'ont-ils aucune valeur définitive, et nous ne les citons pas dans le but d'en tirer des conclusions qui le seraient elles-mêmes. Plus précisément, nous voulons relever les remises en question apportées par le fait que certains acquis disparaissent, alors que d'autres possibilités doivent être envisagées. L'anthropologie théologique ne peut ignorer le fait que ces résultats remettent en question certaines données sur lesquelles elle a posé certaines de ses fondations ${ }^{27}$. Pour la théologie, que signifie la prise au sérieux des recherches récentes et actuelles en sciences de la nature autour de l'hominisation?

\section{Questions émergentes en paléo-anthropologie et en cognition animale}

En paléo-anthropologie, les six dernières années ont apporté des découvertes qui obligent à abandonner la conception linéaire de l'hominisation, comme différenciation progressive d'une souche de

27. Il est exclu d'emblée que lesdites remises en questions soient traitées dans le cadre général, encore repris récemment, par Wesley Wildman, d'une interrogation inquiète sur les utilisations anti-théistes de la science ou sur la légitimité de la théologie en face de la science. Du reste, Wildman lui-même, tout en inscrivant tout son propos sous la rubrique «science vs religion? ", observe du même souffle le caractère marginal de l'utilisation anti-théiste par les scientifiques. W.J. Wildman, «A Theological Challenge: Coordinating Biological, Social, and Religious of Humanity», Zygon, 33/4 (1998) p. 571-597. 
primates par rapport au reste du monde animal. Récemment encore, on pouvait soutenir que l'humain était le résultat de mutations ayant affecté une espèce unique, enchaînement de variations sur le thème d'homo erectus; celui-ci aurait donné naissance à des descendants toujours de plus en plus proches de l'espèce homo sapiens sapiens. Une branche spécifique, unique, aurait ainsi évolué, à côté des autres branches des primates ayant débouché sur ces autres espèces contemporaines que sont, en particulier, les chimpanzés et les deux espèces de gorilles. Or, il apparaît maintenant que les caractères ordinairement conçus comme des indices d'hominisation ont existé chez des espèces auxquelles la nôtre ne serait pas liée; ces traits dits humains se seraient déposés et développés ailleurs que dans notre lignage.

Ainsi, un cas récent incite à une certaine prudence dans la surévaluation des transformations physiologiques découvertes en paléoanthropologie. En l'an 2000, une équipe de chercheurs a mis au jour les restes d'un hominien, contemporain des australopithecus, démontrant certains traits qui le rendent plus "moderne" que ces derniers $^{28}$. En particulier, il possède une tête plus plate que celui-ci, ce qui avait été compris jusqu'alors comme un indicateur d'un degré avancé dans l'hominisation. Or, l'hominien en question est vieux de 3,5 millions d'années. Il n'appartient ni à la famille australopithecus, ni à la famille plus tardive homo; les chercheurs ont donc constitué un nouveau groupe, celui des kenyanthropus platyops. Cet hominien, dont le crâne "moderne" contredit l'âge, remet en question l'idée d'une série linéaire de mutations qui auraient touché une souche unique jusqu'à produire notre espèce. Elle suggère que ce que l'on interprète comme un haut indice d'hominisation ne serait que le résultat d'une adaptation à l'environnement, apparue en une espèce

28. H. GeE, "Un curieux pré-humain, primitif et moderne à la fois », Le Monde (23 mars 2001, p. 25); IDEM, "La mosaïque des origines humaines déconcerte les paléontologues", Le Monde (23 mars 2001) p. 25. Selon le vocabulaire courant en paléo-anthropologie, un hominien est un primate appartenant à la souche générale dont homo erectus est issu, alors qu'un hominidé est un homo erectus ou l'un quelconque de ses descendants. 
puis disparue pour ressurgir ailleurs. Quoiqu'il en soit, kenyanthropus platyops, ainsi que d'autres espèces fossiles récemment découvertes et qui embrouillent les catégories australopithecus ainsi qu'bomo habilis, obligent à reconnaître que l'arbre généalogique des hominiens est singulièrement plus ramifié et complexe que ce qu'on avait cru. Il y aurait eu de l' «humain » ailleurs que chez nos ancêtres. Ici, on ne parle que d'une morphologie crânienne. Mais si la même chose était vraie ailleurs? Dès lors, faudrait-il envisager la possibilité que l'hominisation touche des souches animales différentes? Ou nous demander si certains caractères morphologiques ne seraient pas rangés sous la rubrique «hominisation» qu'en vertu d'une erreur de perception?

\subsection{Homo neandertalensis et homo sapiens sapiens}

Auparavant, les années 1996 et 1997 avaient apporté des résultats de recherches paléo-anthropologiques et génétiques qui concernent un hominidé considéré jusqu'alors comme un très proche ancêtre d'homo sapiens sapiens, l'homme de Neandertal. Des analyses génétiques d'homo neandertalensis par une équipe de chercheurs allemands et américains mettent en évidence l'absence de parenté entre lui et homo sapiens sapiens ${ }^{29}$. Elles démentent ainsi l'idée reçue, voulant que cet hominidé soit l'ancêtre d'homo sapiens (notre parent immédiat). Quoique issu d'homo erectus, comme l'est homo sapiens, les troncs sapiens et neandertalensis se sont séparés à une époque encore indéterminée. Homo neandertalensis a habité l'Europe sans partage jusqu'à l'arrivée des sapiens venus d'Afrique et d'Asie mineure, il y a 40000 ans. Les deux espèces auraient coexisté durant une période de 10000 ans. Elles auraient pu se fréquenter sur une base pacifique, si l'on en croit une équipe de chercheurs du Musée de l'homme, à Paris, qui a décelé dans un site néandertalien des objets de fabrication typiquement sapiens ${ }^{30}$. Aux alentours de 30000

29. Voir A.E. Messer, "DNA Shows Neandertals Were Not Our Ancestors", Penn State Newswire, 7 novembre 1997, http:/www.psu.edu/ur/NEWS/news/ Neandertal.html. 
ans avant notre ère, on perd toute trace de l'homme de Neandertal. A-t-il été déclassé par homo sapiens au jeu de la lutte pour la survie dans un environnement glaciaire ${ }^{31}$ ? Aurait-il été l'objet d'un génocide, perpétré par nos ancêtres? On l'ignore. Ce qui est certain, toutefois, c'est que notre espèce n'est pas le résultat d'une mutation des néandertaliens. D’eux à nous, il n'existe aucun rapport généalogique. Tout au plus peut-on imaginer que la population des quelques néandertaliens restants se soit assimilée à la population des sapiens, pour s'y dissoudre au point de ne plus y laisser la moindre trace génétique.

La dissemblance génétique prouve qu'on a affaire à une souche autre que la nôtre. Or, on ne saurait ignorer les similitudes morphologiques et comportementales entre les deux espèces, similitudes si grandes qu'on verrait mal comment dénier l'humanité à l'homme de Neandertal, ancêtre comme nous d'homo erectus et doté, par surcroît, d'un cerveau plus volumineux que le nôtre. Citons à cet égard une autre recherche récente, pratiquée sur un site belge, qui met en évidence des habiletés techniques insoupçonnées jusqu'alors de la part des néandertaliens, sur des artefacts très antérieurs à l'arrivée des homo sapiens. Elle s'ajoute aux multiples artefacts d'activités culturelles et rituelles de ces hominidés ${ }^{32}$.

30. Voir M. Berkowitz, «Newsbrief: Neandertal News", Archaeology, 49/5 (1996) p. 22.

31. Une autre équipe trouve les restes alimentaires d'un groupe de sapiens se nourissant de petit gibier et de poisson, ce qui serait une différence marquante par rapport à l'homme de Neandertal et un avantage possible dans un contexte de rareté alimentaire et de course aux protéines. Voir H. Morin, «L'homme de Cro-Magnon a mis carpes et lapins dans le moteur de l'évolution », Le Monde (25 mai 2001) p. 19.

32. L'homme de Neandertal aurait souffert d'une dysfonction chronique de la glande thyroïde, caractéristique des homo sapiens sapiens souffrant de crétinisme; en effet, ses caractéristiques morphologiques le rapprochent de ceux-ci (petite taille, corps trapu au squelette massif, arcade sourcilière prononcée, etc.). Voir A.-M. Simard, "La seule petite différence entre nous et l'homme de Neandertal ", Québec-Sciences (novembre 1998). Par contre, voir J.-M. Veszely, "Neandertal: l'énigme de Remicourt», Le Soir Magazine (18 mars 
Dès lors, si par hypothèse on en venait à produire un néandertalien par clonage, il serait probablement hors de question de l'enfermer dans un jardin zoologique ou de le traiter comme un singe de laboratoire. Génétiquement séparé de notre espèce, il pourrait probablement compter sur un appui populaire et scientifique visant à le faire reconnaître comme l'un des nôtres, fut-il plus frustre que nous (moins, toutefois que ce qu'on a longtemps pensé), et comme jouissant des mêmes droits que quiconque. À considérer les choses sur le plan génétique, force est de reconnaître qu'homo sapiens sapiens ne représente pas le point de convergence et de recueillement du groupe bomo.

La correspondance génétique n'est donc pas la pierre de touche de l'humanité (au sens de qualité humaine). Reste l'élément culturel, évoqué à l'instant à propos des néandertaliens. Ceux-ci seraient humains parce que culturellement actifs et dans cette mesure, en plus du fait de leur parenté avec homo erectus. Si l'on suit cette voie, il importe de tenir compte d'une activité culturelle possiblement plus riche chez les néandertaliens que chez les premiers homo sapiens. Ce qui impliquerait une proximité plus grande, pour un temps bref, entre les premiers et nous qu'entre homo sapiens et homo sapiens sapiens, une humanité plus accomplie chez les néandertaliens que chez les premiers représentants de ceux qui sont nos ancêtres immédiats.

Et si cette humanité que l'on reconnaît sans peine chez les néandertaliens n'était qu'un effet de ressemblance morphologique, un effet de perception et de sympathie? La question est troublante: elle blesse le poids que nous accordons à la différence humaine. En outre, elle paraît hautement simplificatrice: la qualité humaine tiendrait à d'autres caractères, plus fondamentaux (dirons-nous provisoirement) que la ressemblance morphologique et physionomique.

1998). Dans le même sens, à propos d'autres fouilles, voir G. Drouin, «À la recherche de Neandertal. Les fouilleurs dégagent le sol anthropique H-1 vieux de 200000 ans", Réseau, 30/6 (mars 1999) p. 8-11, où l'on apprend que certains néandertaliens avaient atteint un niveau de raffinement technologique insoupçonné jusqu'alors. 
En invoquant l'aptitude culturelle, nous les impliquons: le langage et la conscience réflexive.

\subsection{Langage et intelligence réflexive}

L'humain émet des sons porteurs de signification; il les apprend, puis les agence pour produire du sens; il fait montre d'une créativité constante tant dans la formation de nouveaux mots que dans l'agencement des mots qu'il emploie. En plus du langage verbal, il use du langage écrit, ainsi que du langage symbolique. Son rapport au monde est imprégné de langage, si bien que toute réalité humaine porte une dimension langagière. Plus encore: une expérience humaine est a priori langagière. Si bien que là où on rencontre du langage, on trouve de l'humain. "Il n'y a d'homme que parlant", pour reprendre la fameuse expression de Heidegger. En parlant, au sens le plus complet du terme, qui va bien au-delà du fait de produire des sons articulés (un perroquet n'y arrive-t-il pas?), l'humain réalise aussi une autre de ses aptitudes propres: il inscrit sa position existentielle dans le monde, il actualise la conscience qu'il a de luimême, son intelligence réflexive. Aussi considère-t-on l'accession au langage, et la conscience réflexive qui lui est reliée, comme des seuils déterminants pour l'hominisation. Langage et conscience réflexive sont le propre de l'humain. Il n'y a pas de langage sans humain, ni d'humain sans langage.

L'étude de la cognition animale remet en question cette position en cherchant à mesurer les capacités réflexives et langagières de divers animaux. Et il semble que l'observation expérimentale de certains animaux fasse découvrir chez eux des aptitudes langagières qu'on a toujours considérées comme spécifiques à l'humain. Ainsi, les expériences de Irene B. Pepperberg sur un perroquet gris africain ont révélé un animal apparemment capable de beaucoup plus que d'une reproduction mécanique de mots ${ }^{33}$. Pepperberg a commencé

33. Pour une présentation dans un contexte théologique, voir G.R. Peterson, "The Evolution of Consciousness and the Theology of Nature ", Zygon, 34/2 (1999) p. 293-295. 
par enseigner à son perroquet un vocabulaire comportant 30 noms d'objets courants, 7 couleurs, 5 formes et les nombres de deux à six. Par la suite, elle a soumis l'animal à des exercices au termes desquels il a démontré les capacités suivantes: capacité de compter les objets, entre deux et six; formulation de phrases fort simples mais non apprises; réponse à des questions faisant appel à des catégories relativement abstraites: «De quelle couleur est l'objet?» "De quelle forme? " Placé devant un groupe d'objets, il pouvait aussi répondre aux questions: "Qu'est-ce qui est semblable? Qu'est-ce qui est différent?»

Non seulement le perroquet a-t-il pu, avec un pourcentage de réussite de 76,7\%, reproduire des mots, mais il en comprenait la signification et il pouvait les prononcer pour compter, décrire, demander ou répondre à une question à lui posée; en un mot, pour entrer en relation et y inscrire une position "personnelle" (de demandeur, questionneur, répondeur...). Capacité langagière très rudimentaire, certes. Autant que celles de l'enfant de 15 mois qui apprend à nommer les premières réalités matérielles de son environnement.

Qu'on puisse tirer de telles conclusions de recherches menées auprès d'un non-mammifère, doté d'un cerveau aussi rudimentaire que celui d'un perroquet, interroge le seuil de différenciation que nous posons entre notre espèce et le reste du monde animal. Que trouverait-on chez des espèces plus proches de la nôtre? Une autre recherche le suggère, menée par Elizabeth Sue Savage-Rumbaugh sur un bonobo, ou chimpanzé nain ${ }^{34}$. Il est bien connu que d'autres recherches ont déjà été réalisées sur des primates en vue de mesurer leurs capacités à parler. Leurs méthodologies ont souvent été critiquées. Celle de Savage-Rumbaugh a reçu au contraire un accueil largement favorable de la part de la communauté scientifique.

À l'état sauvage, le bonobo adopte souvent la station debout; il est plus intelligent, plus sociable et moins violent que les chimpanzés, et ses pratiques sexuelles intenses et créatives dénotent un sens poussé des échanges sociaux. Il fabrique aussi des outils rudimentaires en

34. Peterson, "The Evolution of Consciousness », p. 295-297. 
pierre, comme nos ancêtres australopithèques. Sur le plan génétique, il s'agit du singe le plus proche de notre espèce.

Il serait long et inutile de reprendre ici dans le détail la teneur des expériences effectuées avec le bonobo de Savage-Rumbaugh, appelé Kanzi, et qui sont rapportées ailleurs ${ }^{35}$. Notons les résultats suivants:

1. L'expérience de Savage-Rumbaugh, qui consistait à tester les capacités d'utilisation par un bonobo d'un langage symbolique, avait d'abord été menée avec un succès mitigé sur la mère de Kanzi. Celui-ci naquit en cours d'expérience et, en assistant à l'entraînement de sa mère et sans que personne ne l'ait entraîné à le faire, se mit spontanément à utiliser le langage symbolique élaboré pour l'expérience, avec un succès beaucoup plus grand que sa mère.

2. Kanzi apprit aussi un autre langage dont les symboles désignaient 17 sites dans une forêt adjacente au laboratoire. Après 16 mois il maîtrisait un langage symbolique composé d'une cinquantaine d'éléments. Il désignait ces sites à distance et sans y avoir été incité. Si on lui présentait un symbole, il se rendait immédiatement au site correspondant. Savage-Rumbaugh en conclut à une rudimentaire capacité de pensée abstraite.

3. Kanzi apprit aussi à combiner deux ou trois symboles pour exprimer une réalité complexe. Il se mit à construire des énoncés que personne ne lui avait appris, pour parvenir à des résultats que personne ne lui avait suggérés. Une linguiste analysa les énoncés de Kanzi, pour établir qu'ils obéissaient à une grammaire, aussi élémentaire fut-elle.

4. On effectua des tests comparatifs entre Kanzi et une fillette d'un an et demi, mesurant leurs degrés respectifs de compréhension de requêtes verbales à trois éléments, du type de: «donne l'objet $a$ à l'individu $x$ », ou encore "pose l'action $a$ sur l'objet $x »$. Ces tests portèrent sur 660 énoncés et s'échelonnèrent sur une pé-

35. Pour une telle description, Peterson, «The Evolution of Consciousness », p. 295298. 
riode de 9 mois. À la fin, on compila les résultats, pour en arriver à une réussite moyenne de $72 \%$ pour Kanzi et de $66 \%$ pour la fillette.

En résumé, en ce qui concerne le langage symbolique, Kanzi démontra les capacités suivantes: compréhension préalable de quelques symboles sans aide humaine; compréhension des symboles préalablement à leur utilisation; utilisation des symboles en l'absence de leurs référents; élargissement remarquable de son "vocabulaire »; utilisation des symboles pour signaler des intentions; agencements originaux des symboles pour construire des énoncés non appris; référence à une grammaire. En ce qui concerne le langage verbal, les résultats de Savage-Rumbaugh montrent aussi certaines aptitudes supérieures à celles de la fillette retenue pour l'expérience.

Les aptitudes relevées chez un bonobo et un perroquet au cours des expériences rapportées ci-dessus démontreraient que des animaux non humains peuvent effectuer des opérations mentales et verbales communément considérées comme les seuils d'hominisation les plus déterminants, parole et conscience de soi comme existant. Or, comme le conclut Peterson,

La question n'est pas de savoir si les singes ont accès au langage mais à quels degrés de développement et de complexité il apparaît et se développe [chez eux]. Le langage et les aptitudes rationnelles n'apparaissent pas «tout d'un bloc" [do not appear whole cloth], mais sont eux-mêmes des combinaisons d'aptitudes plus élémentaires. Parler une langue implique une compréhension (souvent inconsciente) de la représentation, des règles syntaxiques et des unités phonétiques, pour ne citer que quelques éléments. Les patients atteints de dommages cérébraux peuvent souffrir de handicaps très spécifiques tandis que d'autres aptitudes linguistiques demeurent intactes. Par conséquent, il n'est pas étonnant que d'autres animaux peuvent, sous certaines conditions, révéler certains aspects de leurs cerveaux ${ }^{36}$.

Si la thèse de Descartes est exacte, à l'effet que la présence de la raison et du langage indique la présence de la conscience et de la conscience

36. Peterson, «The Evolution of Consciousness», p. 298. 
de soi, Kanzi, comme Alex (le perroquet d'Irene Pepperberg), possède la conscience et, peut-être à la différence d'Alex, la conscience de soi à un certain degré37.

Que doit-on penser, dès lors, ou tout au moins quelles possibilités faut-il envisager, s'il s'avère que ces seuils d'hominisation que sont l'accession au langage et la conscience réflexive peuvent être franchis, ou l'ont été, par des animaux que personne ne songerait à considérer comme humains? Ces études en cognition animale tendent à radicaliser ce que les analyses génétiques des néandertaliens nous suggéraient: non seulement il y aurait de "l'humain" ailleurs que chez les hominidés, mais cela serait vrai même pour des nonhominiens, et même pour des non-mammifères. De plus, on trouverait chez des espèces animales éloignées de la nôtre deux caractères, conscience réflexive et langage, que la tradition anthropologique occidentale invoque pour considérer l'humanité comme un sommet d'évolution animale. Sur le plan du degré de développement de ces caractères, cela est indubitable; mais en chaque humain qui naît, langage et conscience de soi ne sont-ils pas donnés qu'à l'état potentiel? Si le degré de développement de ces aptitudes devait jouer comme critère d'humanité, ne serions-nous pas conduits à considérer nourrissons et handicapés mentaux, voire même muets, comme moins humains que les adultes normalement intelligents et non handicapés $^{38}$ ?

37. Peterson, "The Evolution of Consciousness", p. 298.

38. Par contre, comme le fait remarquer Luc Ferry (dans un ouvrage écrit en collaboration avec le biologiste Jean-Didier Vincent, qui répudie les conclusions de Savage-Rumbaugh), on anticipe la parole du nouveau-né; on n'attend rien de tel du chien ou du bonobo. Le bébé acéphale aurait pu (aurait dû) entrer dans une dynamique intersubjective; le mutisme du cheval n'est pas exceptionnel. Des humains se préoccupent du soin des grands singes, mais ceux-ci se désintéressent du soin des humains. Ferry a partiellement raison: chez l'humain, le sens de la communication relève à certains égards de l'instinct: l'humain veut parler. Ensuite, alors que chez les animaux les rudiments de langage ont une fonction «impérative » (ils ne s'expriment que pour demander à manger, à jouer, etc.), le langage chez l'humain comporte une fonction « déclarative", par laquelle l'humain "commente» le monde et "partage ses connaissances avec autrui ». Voir L. Ferry et J.-D. VInCENT, Qu'est-ce que l'homme? Sur les fondamentaux 


\section{Définir l'hominisation}

De là, une option s'ouvre: ou bien nous étendons le concept d'hominisation à des formes de vie animale passablement éloignées des hominiens, ce qui déboucherait sur sa modification majeure; ou bien nous questionnons non pas l'extension du concept d'hominisation mais sa pertinence elle-même: se nourrirait-il, du moins en partie, de l'erreur de perception commise par une espèce qui se croit la dépositaire exclusive de capacités plus répandues qu'elle ne l'a toujours cru?

Ces deux options font difficulté pour la même raison: le concept d'hominisation, en effet, est essentiel à la nomination de ce groupe de vivants que nous sommes, et dans une option comme dans l'autre, on se retrouverait sans mot pour nous désigner nous-mêmes. Sauf à vouloir classer parmi les faux problèmes celui de la spécificité de notre espèce au sein de la communauté animale, on ne voit pas comment on pourrait juger non pertinent le problème de l'hominisation et de l'humanité, au sens de la qualité humaine. Les deux avenues ci-devant citées sont donc écartées. Reste à définir l'hominisation et l'humanité de manière à tenir compte des options fermées et des possibilités ouvertes par les résultats scientifiques rapportés ici à grands traits. Évoluant dans l'ordre du provisoire qui est la signature de ces résultats, nous proposerons maintenant quelques règles dont le statut ne se veut pas plus définitif que les observations auxquelles elles se rapportent, et qui sont à comprendre comme des principes de méthode en vue d'une anthropologie théologique conséquente. Comment dire l'hominisation d'une manière qui tienne compte des recherches scientifiques exposées ici et des éventualités qu'elles ouvrent?

de la biologie et de la philosophie, troisième édition, Paris, Odile Jacob, 2001, p. 98-99, 169-170. Il n'empêche que les aptitudes langagières du bonobo Kanzi, si sous-humaines soient-elles, ouvrent la question d'une possible gestation du langage à l'échelle de l'évolution, en dehors de l'espèce humaine. Enfin, quant à l'intersubjectivité qui amène les humains à se préoccuper des animaux et non l'inverse, le comportement courant des chiens domestiques envers les jeunes enfants ou envers leurs maîtres malades contredit régulièrement cette assertion. 
Notre thèse est à l'effet que le discours sur l'hominisation est à comprendre sur deux plans, l'un descriptif, l'autre performatif. Il faut prendre la mesure du premier niveau pour passer au second, qui est d'une importance majeure pour le présent exposé.

Sur le plan descriptif, les recherches récentes et en cours en paléo-anthropologie et en cognition animale font l'hypothèse, voire tendent à démontrer la porosité des délimitations entre homo sapiens sapiens et le reste du monde animal. Certains des attributs les plus référés dans la spécification de l'humanité sont recherchés, voire observés chez d'autres espèces. Certes, ils s'y rencontrent à un moindre degré de développement. Mais c'est bien de développement dont il s'agit, c'est-à-dire d'un processus ouvert. Il pourrait concerner les bonobos; il a touché les néandertaliens. Sous cet angle, le discours sur l'hominisation désigne un processus évolutif qui déborde le lignage d'homo sapiens sapiens, mais dont ce dernier est l'espèce la plus aboutie dans la présente période de l'histoire de la vie, quoique de manière aléatoire, contingente et provisoire. Pour parler en termes analogiques et dont on mesure ici le caractère inapproprié - et sans prétendre par là recueillir en une formule tous les aspects du processus qui a produit l'humain -, ce n'est pas uniquement notre espèce qui 's'hominise', mais le règne animal considéré dans son ensemble. Les recherches présentées ici invitent à situer le devenir de notre espèce dans ce contexte élargi, quitte à redessiner les frontières d'un processus d'évolution spécifiquement humain ${ }^{39}$.

39. Comment ne pas évoquer ici, à l'appui de cette position, l'application des sciences cognitives et des mathématiques quantiques à la robotique, ainsi que les résultats troublants qui en résultent? Au laboratoire d'informatique de ParisVI, à l'Université du Sussex au Royaume-Uni, à l'Institut Max-Planck de Münich, à l'Institut fédéral de robotique de Fribourg en Suisse, et ailleurs encore, on fabrique des robots qui apprennent, intègrent des données par euxmêmes et modifient par eux-mêmes leurs comportements en conséquence. Regroupés sur une période prolongée, ces robots programmés différemment ont dû apprendre à cohabiter. Au Computer Science Laboratory de Sony, certains d'entre eux ont développé un langage, qu'ils sont pour l'instant seuls à comprendre, mais au moyen duquel ils interagissent sans interférence humaine. $\mathrm{Au}$ Massachusetts Institute of Technology, on vise maintenant à créer des 
De ce qui précède, il apparaît en outre que la notion d'humanité recouvre un faisceau de caractéristiques variées et variables selon les espèces à caractériser: langage, conscience réflexive, culture, structure génétique spécifique, rapport de descendance à homo habilis et homo erectus, traits de morphologie tels que la station debout, présence de la main, tête plate, etc. On ne saurait fixer l'humanité en un ou deux caractères fixes ${ }^{40}$. On peut trouver chez un représentant de la souche homo telle caractéristique absente chez un autre; ce qu'on ne trouvera pas chez cet autre, on le compensera par telle caractéristique différente. Dans tous les cas, on repérera de l'humain. Ou au contraire, l'existence de l'un ou l'autre caractère chez l'un quelconque des autres primates (la main du gorille, la tête plate du kenianthropus platyops, la découverte d'outils sur des sites d'australopithèques pourtant considérés comme pas encore hominidés) n'aboutira pas nécessairement à leur conférer l'humanité.

Nous passons ainsi sur le plan performatif. Le discours sur l'hominisation est apparu avec la thèse darwinienne sur l'évolution des espèces, qui entend rendre compte d'un état de fait, décrire l'origine des espèces actuelles, parmi lesquelles se trouve la nôtre. Il émerge à une époque précise, ou il trouve ses structures argumentatives, ses logiques sous-jacentes, et à partir d'une position subjective nouvelle, celle du scientifique moderne, qui pose les problèmes en termes d'historicité et d'émergence ${ }^{41}$. Ce n'est pas seulement la thèse de

robots capables d' "émotions », lesquelles influeront sur leurs comportements: attachement aux humains, hostilité, «instinct " grégaire, découragement (qui leur permettrait de se ménager devant l'impossible), mais à terme des émotions non humaines, générées par les robots eux-mêmes, et que les humains n'auraient pas pu concevoir. Voir E. SENDER, "L'invasion des Robo sapiens », Sciences et avenir, $\mathrm{n}^{\circ} 655$ (septembre 2001) p. 40-45.

40. G. Agamben commentant Linné, fait remarquer que l'auteur de la première taxinomie des espèces animales a défini l'être humain par un nom Homo sapiens, qui n'énonce pas une donnée mais un impératif: L'ouvert. De l'homme et de l'animal, trad. par J. Gayraud, Paris, Payot-Rivages, 2002 (italien 1994), p. 44.

41. C'est la thèse centrale de M. Foucault concernant la naissance des sciences humaines en modernité; Les mots et les choses. Une archéologie des sciences humaines, Paris, Gallimard, 1966. 
Darwin qui est neuve à la fin du XIX ${ }^{\mathrm{e}}$ siècle, mais aussi sa manière de penser le problème de l'origine. Une pensée héritière de Darwin perpétue cette manière; développant le problème de l'hominisation, elle produit un acte qui est à penser, et par lequel les sujets de cet acte réalisent un rapport particulier au monde vivant.

Le discours sur l'hominisation constitue un acte de désignation. Sa fonction est de désigner la communauté animale spécifique dont le locuteur fait partie. Il y a humanité là où un animal énonce «nous, l'humanité ». Ainsi, l' «humanité » est un fait identitaire. Le discours sur l'hominisation vise à dire comment l'espèce du locuteur en est venue à se distinguer du reste du règne animal, car il postule que l'humanité émerge dans la mesure où advient cette distinction de facto. À l'encontre des créationnistes d'hier et d'aujourd'hui, qui reprochent aux évolutionnistes de réduire l'humain au statut de singe plus évolué que les autres, le discours sur l'hominisation trace un mouvement de différenciation de l'espèce homo. Ainsi posé, l'acte désignant l'humain repose sur la question du ou des seuils qualitatifs par lesquels notre espèce s'est dégagée de l'animalité pour résulter en cette nouveauté radicale qu'on appelle "l'humain".

Envisagé de cette manière, le discours sur l'hominisation est différenciatoire: il vise à désigner la différence humaine. Les recherches en cours, avec les premiers résultats qu'elles produisent, contraignent à questionner cette approche de l'humanité et à penser aussi l'hominisation sous un mode inclusif. Les traits d'humanité ont des corrélats ailleurs que dans l'espèce homo. Qu'est-ce à dire, sur le plan théologique en particulier, puisque c'est de là que nous partons pour y revenir?

\section{Impact en anthropologie théologique}

L'anthropologie moderne occidentale a problématisé l'hominisation sous le mode de la distinction de l'humain par rapport au reste du monde vivant. Les découvertes les plus récentes s'ajoutent aux plus anciennes pour inviter l'anthropologie à penser l'hominisation sous un mode plus inclusif. Car s'il appert que les critères modernes de définition de l'hominisation recouvrent des processus repérables au- 
delà de notre espèce, la théologie est invitée à penser le devenir de la nature et celui de l'animal en tant que solidaires du devenir humain, et réciproquement. Plus précisément, la tâche à laquelle toutes ces recherches nous renvoient est de désigner les processus évolutifs qui ont façonné les humains modernes et qui se retrouvent aussi ailleurs (émergence d'une conscience de soi, accession aux diverses formes de langage, apparition d'un éthos culturel) non plus en tant que conditions d'émergence de l'espèce homo, mais en tant que devenir de l'animal.

\subsection{L’humain, seuil indépassable dans la spiritualisation de la Création?}

Nonobstant les remarques formulées précédemment, Rahner rejoint Teilhard sur deux points. D'une part, il refuse l'idée que l'émergence de l'humain ne soit que le produit d'un hasard - hasard dont le scientifique n'a que faire, risque-t-il en $1976^{42}$. Sa thèse de l'autotranscendance active le pousse au contraire à voir en l'humain l'effet décisif d'un processus de maturation bien spécifique de l'univers. Ce processus, c'est la spiritualisation: "...l'histoire du cosmos, au fond, est toujours une histoire de l'espritt ${ }^{43}{ }^{\prime}$. Chez Rahner, l'humain constitue le point d'aboutissement d'une histoire où la matière finit par ne plus pouvoir être dissociable de l'esprit; point définitif pour une histoire de l'univers, souligne-t-il. Histoire de l'univers, ajoutons-le, qui obéit à

un principe métaphysique d'économie: la causalité divine transcendante agit dans le déroulement intramondain des phénomènes de la manière la plus discrète et la plus économique, c'est-à-dire là seulement où surgit originellement un terme essentiellement nouveau et irréductible ${ }^{44}$,

42. K. Rahner, Traité fondamental de la foi. Introduction au concept du christianisme, trad. par G. Jarczyk, Paris, Centurion, 1983 (allemand 1976), p. 216.

43. Rahner, Traité fondamental de la foi, p. 219.

44. RAHNER, "Le monogénisme et la théologie», p. 85. 
mais qui agit concrètement, à tel point que les espèces d'hominiens et d'hominidés dont provient l'homo sapiens sapiens ne pouvaient que s'éteindre, une fois terminé leur travail de préparation en vue de notre émergence ${ }^{45}$. Qu'est-ce à dire? Que notre espèce serait indépassable sur le plan de la spiritualisation de la matière? Affirmation hautement hasardeuse dans une perspective évolutionniste, et qui ne semble plus nécessairement impliquée par la pensée du Rahner du Traité fondamental de la foi. En effet, non seulement pour Rahner "à partir de là l'essence de l'homme lui-même peut être vue à l'intérieur d'une conception fondamentale et globale du monde ${ }^{46}$ », mais on peut envisager sous le jour théologique la possibilité d'une autre créature organique, possédant les caractéristiques spirituelles de l'espèce homo. Non pas l'animal, mais un vivant qui peut fort bien occuper la fonction discursive de l'animal: l'extra-terrestre, cet hypothétique autre de l'homo, doté lui aussi de cette subjectivité (dont le présent article envisage la présence actuelle ou future chez l'animal):

Si quelqu'un présuppose (ce qui assurément, pour une pensée qui œuvre à l'intérieur d'un schéma d'évolution, n'est guère vraisemblable en raison de la grandeur irreprésentable du monde) qu'il n'existe que sur notre terre de tels êtres de liberté à complexion spirituelle-corporelle, réalisant par-devant Dieu leur destin, et s'il se représente que cette histoire globale collective de l'humanité vient à cesser en raison d'une catastrophe cosmique ou d'une catastrophe historique commandée par la race humaine, [...] il pourrait alors, bien sûr, penser sans plus la poursuite du cours du monde et de son histoire physique. Mais une telle représentation ne rend pas proprement justice au fait que, en dépit des sciences de la nature qui font autorité, nous n'avons connaissance de la matière que comme élan vers l'esprit, la subjectivité et la liberté, même s'il nous faut admettre que cet élan, avant qu'il ne parvienne au sujet libre, a une durée temporelle très étendue $e^{47}$.

Ici, Rahner sacrifie à l'hypothèse d'une humanité comme fait spirituel unique dans l'histoire de l'univers pour soutenir que même

45. RAHNER, "Le monogénisme et la théologie», p. 85.

46. RAhner, Traité fondamental de la foi, p. 208.

47. RAhner, Traité fondamental de la foi, p. 492. 
en son absence la matière reste «élan vers l'esprit ». Il est à remarquer que le problème qu'il évoque ici ne se poserait pas s'il se trouvait, ailleurs que sur terre, des êtres intelligents et comparables à l'humanité sur ce plan. La référence à l'extra-terrestre, effet d'époque, se trouve aussi chez Peacocke (à propos de l'Incarnation du Verbe, qui touche non seulement l'humanité mais «toute personne sensible, dotée de conscience réflexive et non humaine, qui habiterait éventuellement [d'autres planètes] et qui serait capable de se relier à $\left.\mathrm{Dieu}^{48} »\right)$. Chez Rahner comme chez Peacocke, l'évocation de l'extraterrestre fait appel aux mêmes questionnements identitaires que nous, humains, portons en face de ces animaux auxquels nous ressemblions jadis, qui pourraient un jour nous ressembler à nouveau, et qui nous ressemblent peut-être déjà plus qu'on ne le croit. Dans le cas de Rahner, si l'on considère ce qu'il écrit au sujet de l'humanité en l'élargissant à une éventuelle vie extra-terrestre, au sujet d' "êtres de liberté à complexion spirituelle-corporelle, réalisant par-devant Dieu leur destin ", à la lumière de sa définition de l'humain comme créature où le devenir de l'univers accède à l'esprit, on pourrait en conclure que l'humanité en ce sens théologique ne coïncide pas avec l'espèce homo. Être humain, ce n'est pas être un descendant d'bomo habilis et d'homo erectus. Être humain, c'est accéder à l'esprit. Un processus, on l'a vu, dont l'étude est à l'ordre du jour en cognition animale. On est encore très loin de la capacité théologale, qui va de pair avec une conscience du tout et de l'absolu, et qui est décisive pour la définition de l'humain chez Rahner; mais dans la conscience de l'évolution comme un processus ouvert, la théologie de Rahner prend une résonance inattendue en invitant à saluer en l'animal une possibilité d'humanité.

Sur le plan de la théologie chrétienne, ceci invite à repenser certaines notions importantes voire centrales de la dogmatique. Des théologies récentes s'engagent déjà dans cette voie.

48. «Any sentient, self-conscious, nonhuman persons that inhabited [other planets] who are capable of relating to God. »(PEAcocke, Theology for a Scientific Age, p. 710) 


\subsection{La contingence de l'espèce humaine et le devenir de la Création}

La foi chrétienne est de type historique. Elle raconte un récit de salut. La révélation n'emprunte pas la voie d'une illumination sur le sens ultime de la réalité en soi, mais celle d'une série de narrations qui composent la trame d'une histoire du salut. Sur ce plan, deux tâches théologiques sont requises au regard des recherches récentes, parfois dans le sillage de travaux déjà réalisés. D'une part, délaissant les avenues de la théologie moderne, penser l'histoire du salut comme histoire de la nature, et non seulement de l'humanité; d'une telle histoire de la nature, celle de l'humanité constituerait une composante. Ce qui revient à dire que le salut de l'humain participerait d'un salut plus originel.

L'autre tâche, qui n'irait pas sans incidence sur la précédente, consisterait à intégrer en théologie l'indétermination du processus évolutif. Cette dernière est-elle compatible avec la confession d'un Dieu créateur ${ }^{49}$ ? Les recherches récentes, fidèles à l'évolution générale de la biologie depuis Monod (1972), et à la théorie du chaos, contredisent l'idée d'un plan préétabli et appelant l'humanité comme nécessaire. Bien au contraire: en l'état actuel des choses, l'humanité apparaît comme contingente et comme un complexe de caractéristiques empiriques. Tout cela renforce une idée introduite récemment en anthropologie théologique, comme on l'a vu précédemment: la contingence de l'émergence humaine, avec son corollaire, la possibilité de son extinction. Par contre, demeure l'idée d'un devenir de la nature, auquel participe l'espèce homo pour la durée de son existence.

\section{3. À l'image de Dieu: de l'humain à la Création}

Par ailleurs, en christianisme, l'humain est qualifié comme créé à l'image de Dieu. Selon Moltmann et Peterson, sur le plan biblique cette qualification ne se trouve que dans le premier récit de création

49. Pour un aperçu de la polémique suscitée par cette question, et sur ses incidences théologiques, voir T.M. McNulty, «Evolution and Complexity ", American Catholic Philosophical Quarterly 73/3 (1999) p. 435-448. 
de la Genèse ${ }^{50}$. La tradition chrétienne a donné divers sens à cette qualification. À titre d'exemples, pour Origène, l'image de Dieu réside dans l'homme appelé à devenir saint comme Dieu est saint (Contre Celse, 63). Pour Clément d'Alexandrie, c'est l'esprit humain qui est à l'image de Dieu (Stromates, 5,14). Augustin fait résider l'image dans la raison ( $\mathrm{La}$ Cité de Dieu, 22,24), tandis que Tertullien, auparavant, la voyait dans la liberté morale (Contre Marcion, 2,5). Aujourd'hui, Peterson, à la suite de G. von Rad, y voit une image référant à la responsabilité humaine à l'égard du jardin d'Éden, dont l'être humain a la gérance ${ }^{51}$. Gérance provisoire, et toute récente, devrait-on ajouter, si on la situe dans le cadre du temps géologique. De la variété des interprétations une leçon pourrait être tirée: le lieu précis de l'image de Dieu en l'humain n'a pas à être défini : dire l'humain à l'image de Dieu, c'est en somme énoncer un rapport essentiel entre l'humain et Dieu; un rapport non univoque, non réductible à une caractéristique précise. Peut-être pourrions-nous voir dans l'imago Dei une expression théologique de cette position de l'être humain dans l'ouvert, dans l'«éclaircie de l'Être ${ }^{52} »$. Or, comme le laisse entendre McFague, et comme le développe un peu plus Peterson, c'est toute la nature qu'il serait approprié de penser comme image de Dieu, à des degrés divers selon les espèces, mais de manière non moins pertinente théologiquement si

50. Moltmann, Dieu dans la création, p. 277; Peterson, «The Evolution of Consciousness ", p. 299. En fait, la qualification de l'humain à l'image de Dieu est plus fréquente que Peterson et Moltmann le croient: Gn 9,6: interdit du meurtre au nom du respect dû à celui qui est à l'image de Dieu; 1Co 11,7: parce qu'il est à l'image de Dieu, l'homme [anèr, individu de sexe masculin] doit garder la tête découverte dans l'assemblée; Jc 3,9: critique du fait que «avec la langue, nous maudissons les hommes, qui sont à l'image de Dieu». Sans compter la récurrence d'un thème apparenté, le renouvellement du chrétien à l'image du Christ chez Paul.

51. Peterson, "The Evolution of Consciousness", p. 299.

52. Voir M. Heidegger, Lettre sur l'humanisme, trad. et présenté par R. Munier (Philosophie de l'esprit), Paris, Aubier Montaigne, 1983 (allemand 1947). 
on considère la biosphère dans son ensemble ${ }^{53}$. Au sein de la biosphère, l'humanité peut certes se désigner comme la pointe la plus avancée de cette image qui évolue et tend à la ressemblance, mais c'est d'un état de fait, récent et provisoire, dont il s'agit ${ }^{54}$.

\subsection{L'hominisation en théologie: la nomination d'une communauté de vivants}

Quel sens y a-t-il à réinterpréter ainsi les catégories traditionnelles de l'anthropologie théologique? Ce qui est en question, ce n'est pas leur justesse descriptive; ce n'est pas non plus leur conformité aux sens originels de ces catégories dans la Bible ou dans les sources chrétiennes. Il s'agit plutôt de se situer face aux effets différenciatoires des catégories en questions et de redessiner les limites de la communauté de vivants dont notre espèce s'estime partie prenante. Une réponse possible de la théologie aux recherches récentes concernant l'hominisation ou qui ont des conséquences majeures sur notre manière de concevoir cette dernière serait donc (1) d'assumer la contingence de l'humanité au sens de l'espèce homo, (2) de rattacher celle-ci à une communauté de vivants qui, en cette espèce particulière, se dit dès maintenant son origine, (3) de penser sous un mode inclusif et ouvert, plutôt que différenciatoire, l'émergence de l'esprit dans l'animalité, la nôtre et celle des autres vivants, ces derniers désormais reconnus comme sujets d'un devenir spirituel, autant dire humain. Enjeu de reconnaissance, sur le plan performatif, qui prend en compte ce qui apparaît de plus en plus clairement sur le plan descriptif. Le devenir humain ne peut plus être pensé seulement comme un devenir de distinction au sein du règne animal. L'histoire de la nature englobe notre histoire et y survivra si d'autres espèces relaient la nôtre pour poursuivre l'histoire. La théologie est conviée à penser

53. Ainsi, Peterson trace quelques analogies entre Dieu et la nature : bonté, ordre (en lien avec la rationalité et la justice divines), évolution de la nature vers de plus en plus de conscience.

54. McFague, The Body of God, p. 113; Peterson, "The Evolution of Consciousness ", p. 299-302. 
cette possibilité, retournant ainsi l'apologétique à double tranchant d'un théologien britannique du $\mathrm{XIX}^{\mathrm{e}}$ siècle finissant qui écrivait:

Le darwinisme est apparu et, sous les allures d'un ennemi, il a réalisé le travail d'un ami. Il a procuré à la philosophie et à la religion un bénéfice inestimable, en nous montrant que nous devions choisir entre deux alternatives: ou bien Dieu est présent partout dans la nature, ou bien il n'est nulle part ${ }^{55}$.

Dans ces conditions, y a-t-il encore lieu de parler d'hominisation ? Certainement, mais en gardant à l'esprit qu'on ne désigne ainsi que le processus évolutif d'une espèce particulière, lequel processus participe d'une évolution plus large. Et qu'après Darwin, une tâche théologique s'ouvre, qui consisterait à développer la portée théologique du devenir animal ainsi que ses conséquences pour la théologie du devenir humain.

\section{Conclusion}

Les récentes recherches paléo-anthropologiques et en cognition animale comportent des implications importantes pour l'anthropologie théologique, plus particulièrement à propos de l'émergence de l'humanité au sein du monde animal. Même si les résultats de ces recherches sont provisoires et si chacune d'entre elles est susceptible de discussion, voire même de réfutation, un fait subsiste, que la théologie ne peut pas ignorer: on n'y écarte pas a priori l'hypothèse d'une porosité des frontières entre humanité et animalité. Bien au contraire. Il en est ainsi parce qu'on se meut dans une perspective évolutionniste. Cette dernière étant couramment admise en théologie, on en vient à considérer théologiquement la possibilité d'un processus d' "hominisation" débordant le cadre de l'espèce homo. Il devient pertinent de construire une anthropologie théologique et une théologie de la création qui tiendraient compte de ce processus. C'est ce que nous proposons ici.

55. A. Moore, "The Christian Doctrine of God», dans C. Gore (dir.), Lux Mundi, 5e édition. London, John Murray, 1889, p. 82. 
Tout en saluant dans sa pertinence le projet teilhardien d'une théologie du devenir cosmique, cette anthropologie et cette théologie de la création devraient se garder d'une perspective finaliste ou téléologique, autant que d'une confusion entre des énoncés relatifs à des phénomènes et d'autres qui sont d'ordre métaphysique ou mythologiques. D'autre part, elles pourraient élargir à l'ensemble du monde animal les implications du concept rahnérien d'autotranscendance active, pensé alors sur une échelle de millions d'années. En conformité avec les positions de Moltmann et McFague, l'objectif serait de faire la théologie d'une communauté animale en devenir, communauté de laquelle l'homo sapiens sapiens représente la réalisation la plus spirituelle (au sens rahnérien) à la présente étape de l'histoire de la vie.

Ainsi formulé, cet objectif invite à reprendre deux concepts usuels en théologie, ceux de l'histoire du salut et de l'image de Dieu: il s'agirait de penser la contingence et le caractère aléatoire de l'espèce homo au sein d'une histoire du salut élargie à une histoire théologique de la vie animale; il s'agirait aussi, sans doute à partir des théologies écologiques de la création, de développer une théologie du salut qui prendrait en compte le salut de la création, et en particulier celui des animaux. D'autre part, cette théologie élargirait à l'échelle de la vie animale la portée du concept d'image de Dieu que la Bible applique à l'être humain.

Par ailleurs, dans une perspective performative et non plus descriptive, il n'est pas moins important d'insister sur l'enjeu éthique de nomination et de délimitation de la communauté de vivants à laquelle l'animal parlant se lie quand il énonce «nous, les humains ». Du sein de la communauté restreinte des hominidés, certes singulière par sa spiritualité et sa tenue dans l'éclaircie de l'Être (en ses représentants sapiens sapiens), nous avons voulu formuler ici une théologie inclusive et hospitalière, attentive à ce commun que nous retrouvons de loin en loin dans le regard, le souffle, la plainte et le jeu de «frère animal». 


\title{
RÉSUMÉ
}

L'orientation actuelle des recherches en paléo-anthropologie ainsi qu'en étude de la cognition animale comporte des implications importantes pour l'anthropologie théologique, plus particulièrement à propos de l'émergence de l'humanité au sein du monde animal. Après avoir résumé l'état de la question à propos de l'hominisation en théologie et les contributions en théologie systématique contemporaine concernant la place de l'être humain dans la nature, puis formulé certaines précisions méthodologiques, l'article présente des recherches récentes (19962001) en biologie et en paléo-anthropologie. Ces recherches questionnent l'extension et l'usage du concept d'hominisation, à propos duquel des propositions sont formulées ici à l'usage de la théologie. Enfin, des enjeux théologiques cruciaux se dégagent de l'étude et sont présentés.

\begin{abstract}
Present paleo-anthropological research, and studies of animal cognition have important implications for theological anthropology, particularly in relation to the emergence of human beings in the midst of the animal world. This article summarises research on the issue of hominization in theology, the contribution of contemporary systematic theology concerning the place of human beings in nature, and issues relating to methodology. It also presents recent research in biology (1996-2001) and paleo-anthropology that questions the use and extension of the concept of hominization to propositions formulated for use in theology. Finally, some crucial theological issues are distilled and presented.
\end{abstract}

(C) Revue Théologiques 2002. Tout droit réservé. 